\title{
Picosecond electrical spectroscopy using monolithic GaAs circuits
}

\author{
Y. Konishi, ${ }^{\text {a) }}$ M. Kamegawa, ${ }^{\text {a) }}$ M. Case, R. Yu, M. J. W. Rodwell, and R. A. York \\ Department of Electrical and Computer Engineering, University of California, Santa Barbara, \\ California 93106 \\ D. B. Rutledge \\ Division of Engineering and Applied Science, California Institute of Technology, Pasadena, \\ California 91125
}

(Received 24 April 1992; accepted for publication 29 September 1992)

This article describes an experimental apparatus for free-space $\mathrm{mm}$-wave transmission measurements (spectroscopy). GaAs nonlinear transmission lines and sampling circuits are used as picosecond pulse generators and detectors, with planar monolithic bowtie antennas with associated substrate lenses used as the radiating and receiving elements. The received pulse is $270 \mathrm{mV}$ amplitude and $2.4 \mathrm{ps}$ rise time. Through Fourier transformation of the received pulse, $30-250 \mathrm{GHz}$ measurements are demonstrated with $\leqslant 0.3 \mathrm{~dB}$ (rms) accuracy.

Broadband terahertz (THz) spectroscopy using femtosecond lasers has been demonstrated by several groups. ${ }^{1-3}$ In these systems, antenna-coupled photoconductors illuminated by the pulsed laser generate and detect subpicosecond electromagnetic transients. Through Fourier analysis of the received signal, material amplitude and phase transfer functions can be measured at frequencies as high as $2 \mathrm{THz}$. Nonlinear transmission lines (NLTLs) and NLTL-gated sampling circuits are an alternative solidstate technology for generation and detection of picosecond transients. ${ }^{4,5}$ Combining NLTLs with broadband antennas, picosecond transients can be radiated and detected for spectroscopic measurements. ${ }^{6}$. The resulting apparatus is very compact, and the transmitters and receivers are inexpensive components fabricated on GaAs with a 5 mask process at $3 \mu \mathrm{m}$ device geometries. The large signal amplitude permits rapid data acquisition and direct display of the received signal on an oscilloscope. The system attains $50.3 \mathrm{~dB}$ accuracy (standard deviation) from 30 to 250 $\mathrm{GHz}$. A key system attribute is frequency resolution, demonstrated through measurements at $100 \mathrm{MHz}$ intervals of a mm-wave Bragg filter.

The NLTL is a transmission line periodically loaded with reversed-biased diodes serving as voltage-variable capacitors which introduce a voltage-variable propagation velocity. ${ }^{4}$ The negative-going transitions of a sinusoidal input are compressed into shock waves, and the output is a sawtooth wave form. In on-wafer measurements, less than $1.8 \mathrm{ps}$ fall time and $4.5 \mathrm{~V}$ amplitude wave fronts have been attained. ${ }^{4}$ NLTL-gated sampling circuits attained similar rise times. ${ }^{4}$

The NLTL output is coupled to a broadband bowtie antenna having a frequency-independent far-field radiation pattern and antenna impedance. This antenna permits close integration of the antenna and NLTL, but has a multilobed radiation pattern, resulting in nonuniformity in the collimated beam. The transmitter NLTL (50 $\Omega$ output impedance), fabricated within one electrode plane, drives

\footnotetext{
${ }^{a)}$ On leave from Shimadzu Corporation, 1, Nishinokyo-Kuwabaracho, Nakagyo-ku, Kyoto 604, Japan.
}

the $120^{\circ} \mathrm{C}(52 \Omega)$ antenna, through a coplanar waveguide (CPW) feed [Fig. 1(a)]. Two $100 \Omega$ resistances at the antenna perimeter terminate the antenna at low frequencies. The receiver is a bowtie antenna interfaced to an NLTL-gated sampling circuit [Fig. 1(b)]. The $0.9 \mathrm{~mm}$ radial lengths of the transmitter and receiver antennas sets a $35 \mathrm{GHz}$ antenna low-frequency cutoff.

To couple the radiated power from the GaAs substrate $\left(\epsilon_{r}=13\right)$, the transmitter and receivers are placed on silicon $\left(\epsilon_{r}=11.8\right)$ hyperhemispherical substrate lenses ${ }^{7,8}$ (Fig. 2). The lenses also partially collimate the antenna radiation. The radiated beam is collimated with off-axis parabolic mirrors, and is focused on the receiver through similar optics. Above the low-frequency cutoff, the antenna system loss is $20 \mathrm{~dB}$ as determined by $45 \mathrm{MHz}-40 \mathrm{GHz}$ network analysis ${ }^{4}$ on larger $(3 \mathrm{~mm})$ antennas. Imaging the

(a)

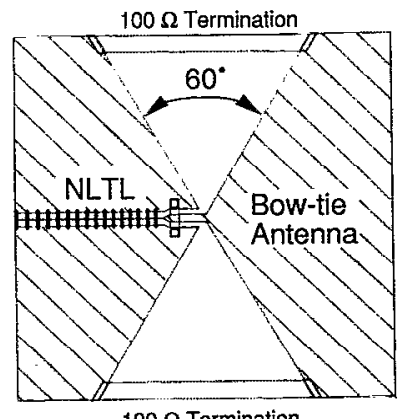

(b)

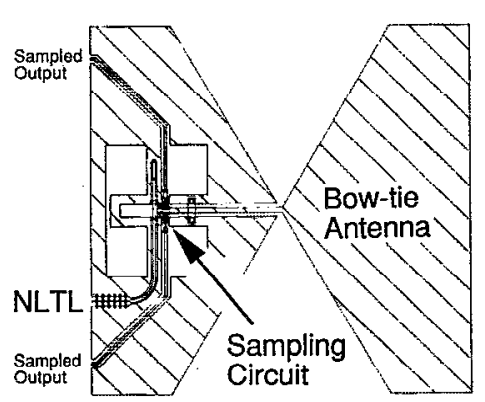

FIG. 1. Simplified integrated circuit layouts of (a) picosecond transmitter and (b) picosecond receiver. 


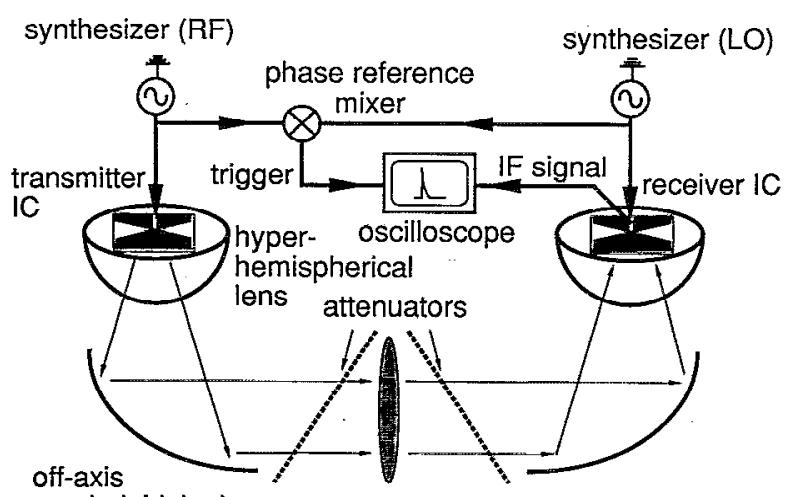

paraboloidal mirror material under test

FIG. 2. Measurement system schematic diagram (left: transmitter, right: receiver). A mixer is used to generate a stable phase reference signal.

transmitter antenna onto the receiver produces a resonant cavity because of reflections at the air-lens and lensantenna interfaces. This cavity is resonant at the frequencies $f_{N}=N c / 2 l$, where $l$ is the transmitter-receiver separation $(l=25 \mathrm{~cm})$. These resonances are suppressed by placing $5 \mathrm{~dB}$ attenuators at oblique incidence on both sides of the sample under test. The effect of cavity resonances on transmission measurements can also be suppressed ${ }^{2}$ by limiting the duration of observation of the received signal. With proper adjustment of the transmitter-receiver spacing, secondary signals arising from multiple transits of the resonant cavity will arrive at the receiver outside the period of observation, and are not mcasurcd. Restricting the period of observation of the received signal to duration $\Delta T$ restricts the frequency resolution of the measurement to $\Delta f=1 / \Delta T,{ }^{2}$ if $\Delta T$ is less than the repetition period of the stimulus signal.

The transmitter NLTL is driven between 7 and 14 $\mathrm{GHz}$, while the sampling circuit is driven at a frequency $100 \mathrm{~Hz}$ below the transmitter frequency. The resulting sampled $100 \mathrm{~Hz}$ signal is observed directly on an oscilloscope. Without the attenuators, the received signal at the sampling circuit output (Fig. 3) is a $270 \mathrm{mV}$ peak-peak pulse train with $2.4 \mathrm{ps}$ rise time $(10 \%-90 \%)$ and a decay time set by the antenna $35 \mathrm{GHz}$ low-frequency cutoff. Res-

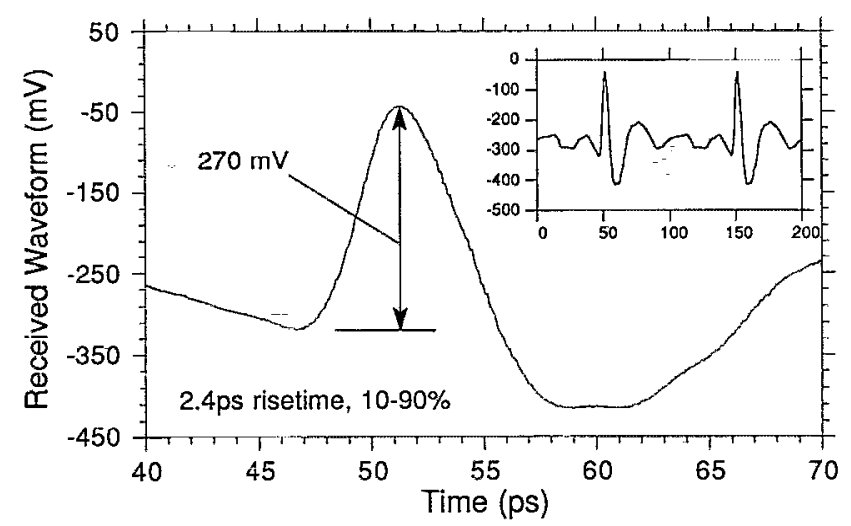

FIG. 3. Received wave form at the sampling circuit output. In the inset the full period of the signal is shown.

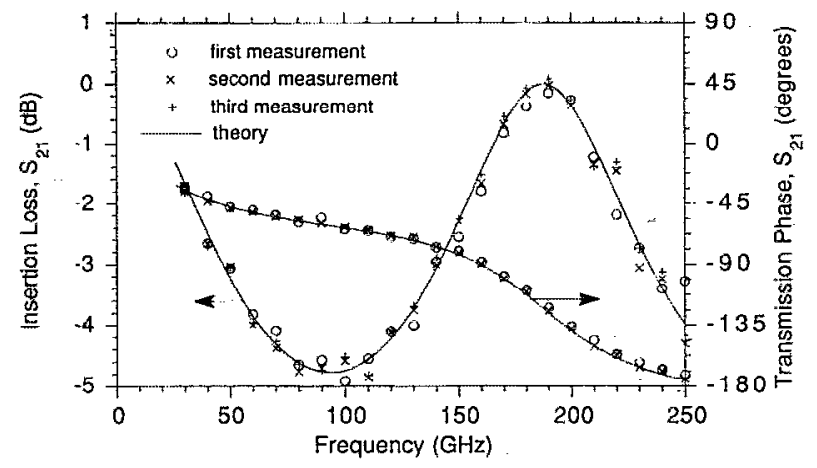

FIG. 4. Vector transmission measurement of a $254 \mu \mathrm{m}$ thickness alumina test sample.

onances observed after the pulse may be arising from reflections within the lens system, the antennas, or the NLTL and sampling circuit.

Attenuation-frequency and phase-frequency measurements are obtained by taking the ratio of the received Fourier spectrum with the device under test in place with the spectrum of a reference measurement taken with the device under test removed. Figure 4 shows the measured transmissivity of a 254- $\mu$ m-thickness $\mathrm{Al}_{2} \mathrm{O}_{3}$ substrate, which has minima when $(\lambda / 4+n \lambda / 2) / \sqrt{\epsilon_{r}}=254 \mu \mathrm{m}$. With three measurements, the accuracy is $\leqslant 0.3 \mathrm{~dB}$ in amplitude and $5^{\circ}$ in phase (standard deviations). Figure 5 shows measured transmission of a Bragg filter consisting of alternating layers of alumina ( 10 pieces, $257 \mu \mathrm{m}$ thickness) and Teflon (9 pieces, $523 \mu \mathrm{m}$ thickness). Data was taken at 100 NLTL drive frequencies between 10 and $11 \mathrm{GHz}$. yielding measurement at the frequencies $n(10 \mathrm{GHz}+m \times 10 \mathrm{MHz})$ where $n$ and $0 \leqslant m \leqslant 99$ are integers, corresponding to 100 $\mathrm{MHz}$ separation at $100 \mathrm{GHz}$. The filter shows $10 \mathrm{~dB} / \mathrm{GHz}$ slope at $118 \mathrm{GHz}$.

We have demonstrated a simple apparatus for accurate, high resolution broadband $\mathrm{mm}$-wave electromagnetic gain-frequency measurements. The current system will allow convenient and accurate measurement of mm-wave materials and components.

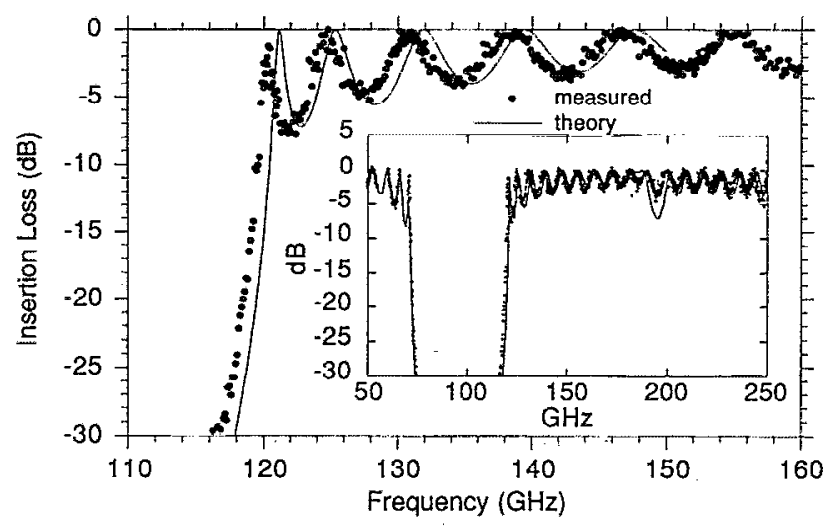

FIG. 5. Transmission measurement of a Bragg filter consisting of alternating layers of 10 pieces of alumina and 9 pieces of Teflon. The inset shows transmission over an expanded frequency range. 
This work was supported by the Air Force Office of Scientific Research under Grant No. (AFSOR-89-0394).

'P. R. Smith, D. H. Auston, and M. C. Nuss, IEEE J. Quantum Electron. QE-24, 255 (1988).

2G. Arjavalingam, Y. Pastrol, J. M. Halbout, and G. V. Kopcsay, IEEE Trans. MTT 38, 615 (1990).

${ }^{3}$ M. Van Exter and D. R. Grischkowsky, IEEE Trans. MTT 38, 1681 (1990).

${ }^{4}$ M. J. W. Rodwell, M. Kamegawa, R. Yu, M. Case, E. Carman, and K. S. Giboney, IEEE Trans. MTT, I194 (1991).
${ }^{5}$ R. A. Marsland, C. J. Madden, D. W. Van Der Weide, M. S. Shakouri, and D. M. Bloom, IEEE GaAs IC Symposium, New Orleans, LA, October 7-10, 1990 (unpublished).

"M. Kamegawa, Y. Konishi, M. Case, R. Yu, and M. J. W. Rodwell, I.EOS Summer Topical Meetings, Newport Beach, July, 24-26, 1991 (unpublished).

${ }^{7}$ D. B. Rutledge, D. P. Neikirk, and D. P. Kasilingam, in Infrared and Millimeter Waves, edited by K. J. Button (Academic, New York, 1984), Vol. 10, pp. 1-90.

${ }^{8}$ R. C. Compton, R. C. McPhedran, Z. P. Popovic, G. M. Rebeiz, P. P. Tong, and D. B. Rutledge, IEEE Trans. Antenna Propag., 622 (1987). 